\title{
ANÁLISE DA MORTALIDADE EM IDOSOS COM COVID-19 NO AMAPÁ ENTRE ABRIL E JUNHO DE 2020
}

Maria Luiza Monteiro Cordeiro; Universidade Federal do Amapá; Marialuizamonteirocordeiro1@gmail.com

Gabriella de Brito Malcher Melo; Universidade Federal do Amapá; gabriellabmmelo@gmail.com Ríllari Oliveira do Nascimento Gomes; Universidade Federal do Amapá; rillari.oliveira.98@gmail.com Thaíla Soares da Costa Picanço; Universidade Federal do Amapá; thailapicanco@bol.com.br

\section{RESUMO}

Introdução: No fim de 2019, uma doença causada pelo vírus SARS-CoV-2 proliferou-se pelo mundo, afetando especialmente os idosos. No Brasil, o primeiro caso foi confirmado em 26 de fevereiro de 2020, chegando ao Estado do Amapá em 20 de março. Objetivo: Analisar a taxa de mortalidade em idosos com COVID-19 e principais comorbidades associadas, no período de abril a junho de 2020. Métodos: Estudo epidemiológico, descritivo, retrospectivo, a partir da análise estatística dos boletins epidemiológicos sobre COVID-19 da Superintendência de Vigilância em Saúde do Amapá (https://svs.portal.ap.gov.br/publicacoes), lançados entre abril e junho de 2020, observando-se faixa etária dos óbitos e comorbidades relacionadas. Resultados: Observou-se, no período analisado, o registro de 405 óbitos por COVID-19 no Estado do Amapá. Destes, 249 (61,48\%) ocorreram em idosos, sendo $98(39,35 \%)$ pertencentes à faixa etária de 70 a 79 anos. Em relação às comorbidades, $79,91 \%$ dos idosos que faleceram por COVID-19 nesse período tinham um ou mais fatores de risco. Hipertensão arterial $(43,71 \%)$, diabetes $(30,15 \%)$, doença renal $(7,53 \%)$ e cardiopatias $(6,03 \%)$ foram as principais doenças crônicas associadas ao óbito. Conclusão: Evidencia-se que a população idosa apresenta maior taxa de mortalidade, devido às comorbidades relativamente comuns associadas à idade, notadamente na faixa de 70 a 79 anos. Hipertensão arterial e diabetes foram os principais fatores de risco, correspondendo a 73,86\% dos óbitos. Considerando maior vulnerabilidade à doença dessa população, o sistema de saúde deve garantir o cuidado e a assistência dos pacientes idosos acometidos pela COVID-19, provendo maior atenção aos portadores de patologias crônicas.

Palavras-chave: Epidemiologia; Covid-19; Idoso. 